\title{
Avaliação da cristalização de mel utilizando parâmetros de cor e atividade de água
} Evaluation of honey crystallization from the colour and water activity parameters

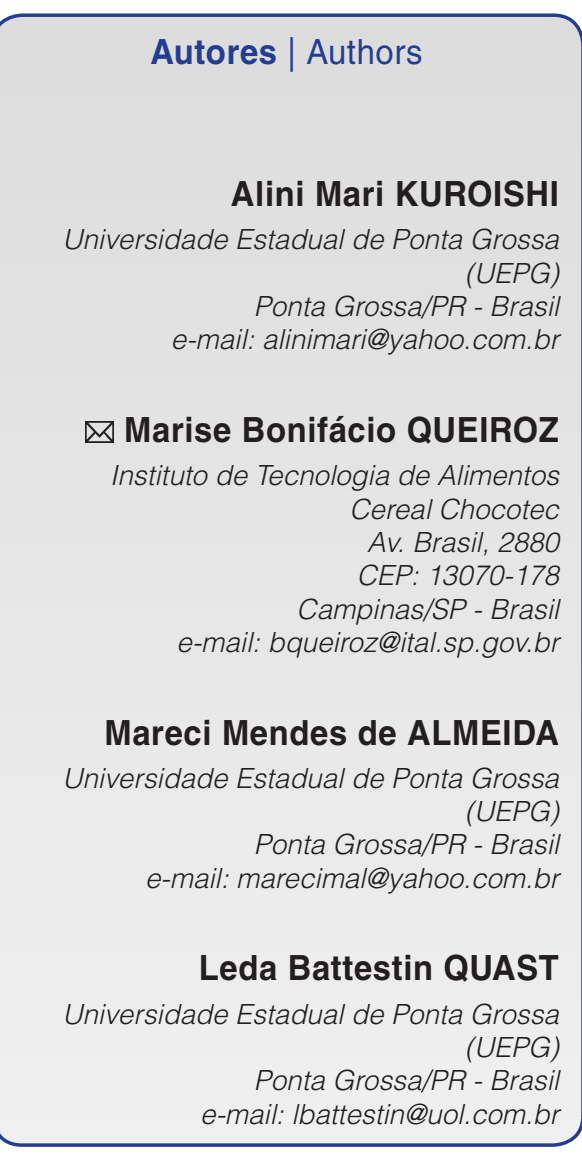

$\triangle$ Autor Correspondente / Corresponding Author

Recebido / Received: 16/02/2009 Aprovado / Approved: 04/10/2011 Publicado / Published: mar./2012

\section{Resumo}

O mel é um dos edulcorantes mais antigos utilizados pelo homem, sendo composto principalmente de monossacarídeos, como a glicose e a frutose, responsáveis em parte pela sua cristalização. A cristalização varia segundo diversos fatores, como a concentração de açúcares, o teor de água na sua composição natural, a procedência floral do néctar, o manuseio durante seu processamento, bem como as condições de estocagem. O presente trabalho teve como objetivo avaliar o efeito da temperatura e da incorporação de cristais de mel na cristalização do mel utilizando como parâmetro de acompanhamento a cor, por meio da determinação do Índice de Brancura (IB) e variação da luminosidade $\left(\Delta \mathrm{L}^{*}\right)$, e a atividade de água. Utilizou-se um delineamento experimental fatorial $2^{2}$, tendo-se como variáveis o percentual de cristais de mel adicionado e a temperatura de armazenamento. Apenas as amostras armazenadas à temperatura de 11 e $21^{\circ} \mathrm{C}$ cristalizaram, sendo mais evidente a cristalização da amostra armazenada em $11^{\circ} \mathrm{C}$, que foi adicionada de $5 \%$ de cristais de mel. O aumento da atividade de água e o aumento no Índice de Brancura (IB), assim como o $\Delta L^{*}$, foram correlacionados com a cristalização das amostras. A maior variação obtida para os valores de $\Delta \mathrm{L}^{*}$ encontram-se na região de temperatura de armazenamento de $11^{\circ} \mathrm{C}$, na qual as amostras cristalizaram mais rapidamente.

Palavras-chave: Mel; Cristalização; Cor; Temperatura; Atividade de água.

\section{Summary}

Honey is one of the oldest sweeteners used by man, composed mainly of monosaccharides such as glucose and fructose, partially responsible for its crystallization. Honey crystallization depends on several factors, such as the sugar concentrations, natural percentage of water, the floral origin of the nectar, handling during processing and the storage conditions. The present work aimed to evaluate the effect of temperature and of the incorporation of honey crystals on honey crystallization, using the colour (as determined by the Whiteness Index - WI and changes in the brightness $-\Delta \mathrm{L}^{*}$ and the water activity as the parameters to accompany the changes. A $2^{2}$ factorial experimental design was used, the variables being: percentage of honey crystals added and storage temperature. The samples stored at 11 and $21^{\circ} \mathrm{C}$ were shown to crystallize, this being more evident in the sample stored at $11^{\circ} \mathrm{C}$ with the addition of $5 \%$ honey crystals. The increase in water activity and in the Whiteness Index, as well as in the $\Delta \mathrm{L}^{*}$ were correlated with crystallization of the samples. The luminosity parameter $L^{*}$ and the Whiteness Index (WI) were higher at the temperature of $11^{\circ} \mathrm{C}$, where the samples crystallized faster.

Key words: Honey; Crystallization; Colour; Temperature; Water activity. 


\section{Introdução}

O mel é um produto alimentício produzido por abelhas melíferas a partir do néctar das flores ou das secreções precedentes de partes vivas de plantas, ou ainda de excreções de insetos sugadores de plantas que ficam sobre partes vivas de plantas. As abelhas recolhem esses materiais, transformam, combinam com substâncias específicas próprias, armazenam e deixam maturar nos favos da colmeia (BRASIL, 2009). Sua qualidade nutricional se deve à presença de vitaminas e minerais, e ao seu elevado valor energético. Somado a isso, o mel apresenta propriedades medicinais, como ação antioxidante e atividade antisséptica, relacionadas à presença de compostos fenólicos (ZUMLA e LULAT, 1989; MOREIRA e DE MARIA, 2001).

A composição exata do mel, assim como os aspectos de cor, aroma e sabor, variam conforme a região geográfica, as condições climáticas e, principalmente, com a florada usada pelas abelhas (SILVA et al., 2004; WIESE, 1986). Apesar dessa variação, o mel é composto principalmente de açúcares, sendo os monossacarídeos, frutose e glicose, os mais abundantes (aproximadamente 80-85\% dos sólidos no mel). Por outro lado, pequenas quantidades de dissacarídeos (maltose e sacarose) e oligossacarídeos também estão presentes (ZAMORA e CHIRIFE, 2006).

O Brasil ocupa a $11^{a}$ posição mundial na produção de mel, sendo a China a maior produtora. Do total de mel produzido no Brasil em 2006, cerca de 40,3\% foi exportado, sendo os Estados Unidos o principal comprador (IBGE, 2006). Segundo o último senso, realizado em 2009, a produção brasileira de mel ficou em torno de 38 mil t (IBGE, 2009), apresentando um crescimento de $14,86 \%$ entre 2006 e 2009.

O consumo de mel no Brasil está estimado em aproximadamente 200 g.pessoa ${ }^{-1}$. ano ${ }^{-1}$, quantidade considerada baixa se comparada a alguns países da Europa, como a Alemanha e a Suíça, onde se calcula um consumo de 2.400 g.pessoa $^{-1}$. ano-1 (MAGALHÃES, 2008).

O conteúdo de água no mel é uma das características mais importantes, influenciando diretamente os seguintes aspectos: viscosidade, peso específico, maturidade, cristalização, sabor, conservação e palatabilidade. A água presente no mel apresenta forte interação com as moléculas dos açúcares, diminuindo a disponibilidade das moléculas de água para o desenvolvimento microbiológico (ABRAMOVIC et al., 2008). A umidade no mel normalmente varia de 16 a $18 \%$, sendo permitido, por legislação, um máximo de 20\% (BRASIL, 2009).

O teor de umidade do mel e sua atividade de água (Aw) são os principais fatores que influenciam a preservação da qualidade do produto, assim como as condições de sua estocagem (ABU-JDAYIL et al., 2002; LAZARIDOU et al., 2004).

As moléculas de água no mel encontram-se ligadas aos açúcares, principalmente por pontes de hidrogênio. No mel líquido, a glicose encontra-se ligada a cinco moléculas de água, enquanto que, no mel cristalizado, a glicose encontra-se ligada a apenas uma dessas moléculas, sendo que as outras liberadas aumentam a atividade de água.

Quando a cristalização ocorre de maneira indesejada e incontrolada durante a estocagem, pode levar à formação de um produto sobrenadante de coloração escura com cristalização desuniforme. A separação de fases forma uma fase cristalina no fundo $e$ uma fase líquida no topo, tornando o mel menos atrativo para o consumidor. A camada do topo contém elevado conteúdo de água, aumentando o risco de degradação do mel por fermentação (GLEITER et al., 2006; ZAMORA e CHIRIFE, 2006).

Por outro lado, quando a cristalização ocorre de maneira controlada, é possível obter produtos como o mel cremoso, no qual há um grande número de cristais muito pequenos que não são percebidos pelo paladar (ESCOBEDO et al., 2006; SUBRAMANIAN et al., 2007).

Todos os méis são líquidos quando produzidos pelas abelhas, mas, por serem soluções supersaturadas de glicose, quando armazenadas em temperaturas abaixo da temperatura média da colmeia $\left(37^{\circ} \mathrm{C}\right)$, tendem a cristalizar espontaneamente, fenômeno esse que não altera a sua natureza (ZAMORA e CHIRIFE, 2006; WIESE, 1986). Essa cristalização varia de acordo com vários fatores, como a concentração de açúcares, o teor de água na sua composição natural, a procedência floral do néctar, o manuseio durante seu processamento, bem como as condições de estocagem (CONFORTI et al., 2006; LUPANO, 1997). Alguns méis nunca cristalizam, outros cristalizam de maneira lenta, como o mel proveniente da flor de assapeixe, ou de maneira rápida, como o mel da flor de nabo forrageiro. A cristalização do mel consiste na separação da glicose, que é menos solúvel que a frutose, e consequente formação de hidratos de glicose. Quando o mel cristaliza, observam-se mudanças na sua coloração (LENGLER, 2008; WIESE, 1986).

Grunennvaldt (2009) relata a utilização da cor como forma de medir a ocorrência de cristalização de gordura na superfície de chocolate (bloom) ao longo do tempo. $O$ autor determina o Índice de Brancura (IB) que relaciona os parâmetros de $L^{*}, a^{*}$ e $b^{*}$ do sistema CIELAB. Seus resultados indicaram uma boa correlação entre o Índice de Brancura e a cristalização da gordura na superfície do chocolate.

Romão et al. (2006) descrevem a avaliação da cristalização em balas duras usando análise de imagem. 
Avaliação da cristalização de mel utilizando parâmetros de cor e atividade de água

KUROISHI, A. M. et al.

Segundo os autores, uma indicação visual de que a bala estaria cristalizada seria a alteração de cor superficial do produto. Acrescentam que a avaliação em conjunto da cor e do halo de cristalização empregando imagens digitais mostrou-se uma ferramenta adequada para acompanhar o processo de cristalização em balas duras, durante a vida de prateleira do produto.

O presente trabalho teve como objetivo propor a utilização da análise de cor (IB, $\Delta \mathrm{L}^{*}$ ) e da atividade de água (Aw) para a avaliação instrumental da cristalização de mel em amostra submetida a diferentes condições de temperatura e diferentes concentrações de cristais de mel adicionados.

\section{Material e métodos}

\subsection{Matérias-primas}

Foi utilizado mel de abelha (Apis mellifera) silvestre com predominância da florada de Capixingui, de dezembro de 2007, colhido na região de Itapira-SP, divisa com Jacutinga-MG. Os cristais de mel sem nenhum tipo de pré-tratamento foram obtidos de um mel de laranjeira em estádio avançado de cristalização, proveniente da cidade de Castro-PR e utilizado previamente em estudos realizados por Vargas (2006).

\subsection{Metodologia}

Para o estudo, utilizou-se planejamento experimental estatístico fatorial $2^{2}$ com 4 repetições no ponto central, com variação do percentual de cristais adicionado na amostra de mel e da temperatura de armazenamento, conforme apresentado na Tabela 1.

Para o preparo de cada ensaio, a massa de mel foi aquecida em um banho termostático (Brookfield, TC-500) a uma temperatura de $40^{\circ} \mathrm{C}$. Os cristais foram adicionados na amostra com auxílio de um agitador mecânico digital (Tecnal, TE-0,39/1) com velocidade de $30 \mathrm{rpm}$, por um período de $15 \mathrm{~min}$. As amostras com os diferentes teores de cristal de mel foram transferidas para frascos de vidro hermeticamente fechados e armazenadas em estufas do tipo BOD por um período de três semanas. A Tabela 1 apresenta as condições do planejamento experimental para o estudo.

\subsection{Determinação do teor de umidade do mel}

A determinação do teor de umidade do mel foi feita de maneira indireta, por meio do método refratométrico utilizado por Conforti et al. (2006). O índice de refração foi medido a $20^{\circ} \mathrm{C}$ utilizando-se um refratômetro digital (Acatec, RDA 8600). A umidade correspondente foi calculada utilizando-se a Equação 1, baseada na regressão linear mostrada na Figura 1. A determinação da umidade da amostra de mel líquido foi realizada em oito repetições.

$$
y=-396,14 x+608,87, \text { com } R^{2}=0,999
$$

\subsection{Avaliação da cristalização}

Para o acompanhamento da cristalização, foram realizadas determinações de atividade de água (Aw) e cor (IB e $\left.\Delta \mathrm{L}^{*}\right)$.

\subsubsection{Atividade de água}

A atividade de água foi medida por meio de um analisador de atividade de água - DECAGON (Aqualab, CX-2) - na faixa de temperatura de 24,7 a $25,3{ }^{\circ} \mathrm{C}$

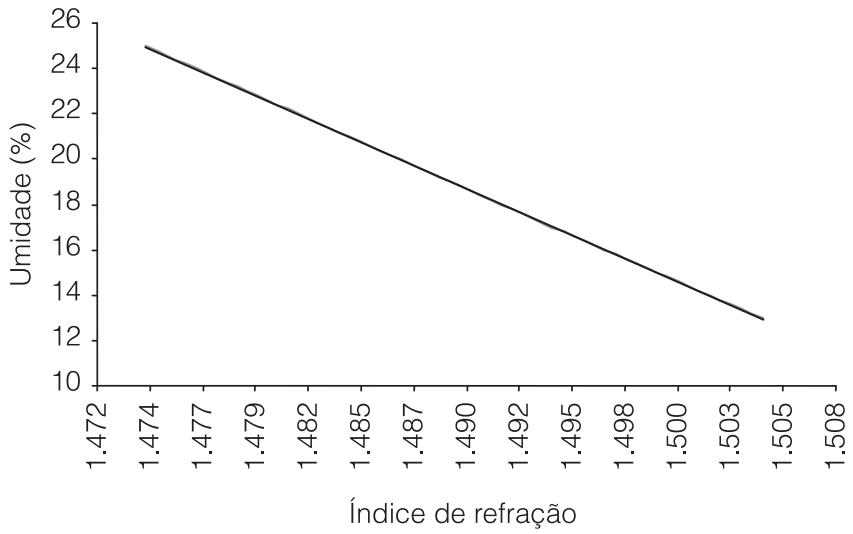

Figura 1. Correlação entre umidade e índice de refração.

Tabela 1. Temperatura de armazenamento e porcentagem de cristais de mel utilizados em cada ensaio

\begin{tabular}{|c|c|c|c|c|}
\hline \multirow{2}{*}{ Ensaios } & \multicolumn{2}{|c|}{ Variáveis codificadas } & \multicolumn{2}{|c|}{ Variáveis reais } \\
\hline & $x_{1}$ & $x_{2}$ & Temperatura $\left({ }^{\circ} \mathbf{C}\right)$ & $\%$ Cristal \\
\hline 1 & +1 & +1 & 31 & 5 \\
\hline 2 & +1 & -1 & 31 & 0 \\
\hline 3 & -1 & +1 & 11 & 5 \\
\hline 4 & -1 & -1 & 11 & 0 \\
\hline 5 & 0 & 0 & 21 & 2,5 \\
\hline 6 & 0 & 0 & 21 & 2,5 \\
\hline 7 & 0 & 0 & 21 & 2,5 \\
\hline 8 & 0 & 0 & 21 & 2,5 \\
\hline
\end{tabular}


(ZAMORA e CHIRIFE, 2006). As análises foram realizadas em seis repetições para cada ensaio.

\subsubsection{Cor}

A cor de cada ensaio foi determinada por meio de um colorímetro (Konica Minolta, CR-410). As leituras foram realizadas no sistema CIELAB $\left(L^{*}, a^{*} e b^{*}\right)$, no qual $L^{*}$ é o componente de claridade ou luminosidade, que varia de 0 a 100, e a* e b* são dois componentes de cor, cuja taxa varia de -100 a 100 ( $a^{*}$ varia de verde a vermelho e $b^{*}$ de azul a amarelo). Para cada ensaio, foram realizadas dez leituras. Antes da realização de cada análise, efetuou-se a calibração do aparelho.

Para verificar a influência da temperatura e do percentual de cristais de mel foi tomada como referência a variação do parâmetro de luminosidade $\Delta \mathrm{L}^{*}$. $\mathrm{O} \Delta \mathrm{L}^{*}$ foi obtido pela diferença entre $0 L^{*}$ no tempo inicial de armazenamento e no tempo de três semanas de armazenamento, sendo, então, $\Delta L^{*}=L^{*}{ }_{\text {zero }}-L^{*}{ }_{3 \text { semanas }}$

Também foi utilizada a evolução do Índice de Brancura (IB) com o tempo de armazenamento. Esse índice é uma conversão dos parâmetros de $L^{*}$, $a^{*} e b^{*}$, sendo calculado segundo a Equação 2 (GRUNENNVALDT, 2009).

$I B=100-\left[\left(100-L^{*}\right)^{2}+a^{* 2}+b^{* 2}\right]^{0,5}$

\section{Resultados e discussão}

O teor médio de umidade do mel foi de $17,55 \% \pm 0,42$ e encontra-se em concordância com a legislação IN no 11(BRASIL, 2009), que determina uma umidade máxima de $20 \%$ e indica que esse mel foi colhido no estádio adequado de maturação. O teor de umidade do mel utilizado neste trabalho está muito próximo aos resultados obtidos por Abramovic et al. (2008), Costa e Pereira (2002) e Silva et al. (2004).

Segundo Escobedo et al. (2006), a umidade inicial do mel é um dos fatores influentes na sua cristalização, pois quanto menor a umidade maior a concentração de monossacarídeos (glicose e frutose), resultando em um maior nível de saturação e, consequentemente, maior probabilidade de cristalizar.

De acordo com Abramovic et al. (2008), durante a cristalização ocorre um aumento na atividade de água, enquanto o conteúdo de umidade se mantém constante.

\subsection{Atividade de água}

A Figura 2 apresenta os resultados da atividade de água das amostras de mel durante o armazenamento. A Média $P C$ representa os valores da atividade de água calculados como a média para os quatro ensaios do ponto central do delineamento experimental.
A atividade de água inicial nas amostras de mel variou de 0,563 a 0,576, concordando com a variação de 0,52 a 0,71 obtida por Conforti et al. (2006) em 148 amostras de mel de Buenos Aires; também em concordância com a variação de 0,483 a 0,591, obtida por Abramovic et al. (2008) em 150 amostras de mel da Eslovênia.

Com exceção das amostras armazenadas a $31^{\circ} \mathrm{C}$ (Ensaios 1 e 2), é possível observar uma tendência do aumento da atividade de água com o passar do tempo. Este resultado sugere que em temperaturas menores (21 e $11^{\circ} \mathrm{C}$ ), a cristalização é favorecida, ocasionando um aumento da atividade de água. Isso pode ocorrer durante a cristalização, porque partes dos sólidos solubilizados no sistema se agregam na forma de cristais, liberando água e tornando a fase líquida menos concentrada, o que faz aumentar a atividade de água. O mesmo comportamento foi observado por Zamora e Chirife (2006) em 49 amostras de méis da Argentina, bem como por Gleiter et al. (2006), que avaliaram 249 amostras de diferentes tipos de mel, e Lazaridou et al. (2004) em um estudo com 33 méis gregos.

Ao final das três semanas de armazenamento, o maior valor de atividade de água obtido foi de 0,611, o qual, do ponto de vista microbiológico, não favorece o desenvolvimento de mofos, bolores, leveduras e bactérias no mel.

\subsection{Cor}

A Figura 3 mostra os resultados para o Índice de Brancura dos méis armazenados nas diferentes temperaturas durante as três semanas e sua evolução com o tempo.

Analisando-se a Figura 3, pode-se observar que o Índice de Brancura permaneceu praticamente constante para as amostras armazenadas a $31{ }^{\circ} \mathrm{C}$ (Ensaios 1 e 2). Nessas amostras, observou-se que durante 0

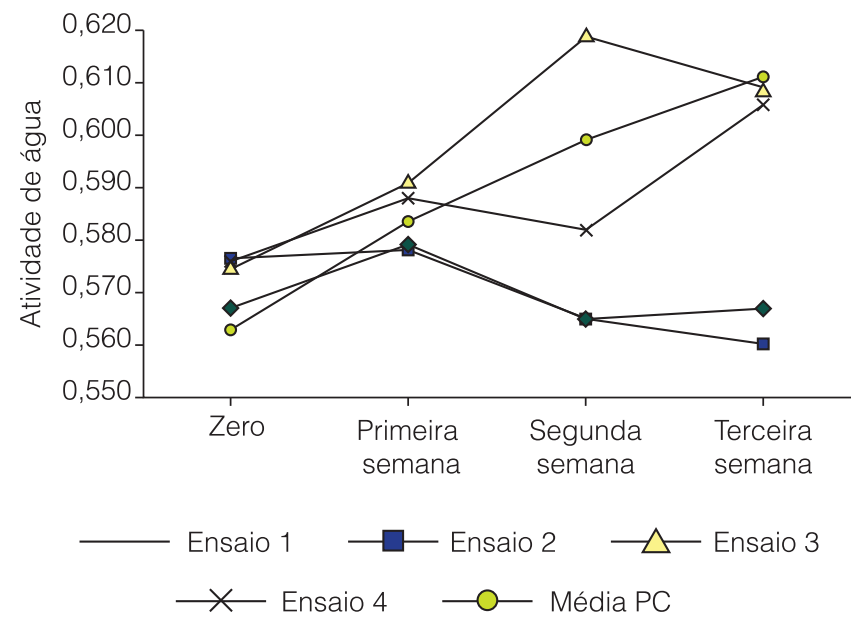

Figura 2. Resultados para a atividade de água para os ensaios do experimento. 
Avaliação da cristalização de mel utilizando parâmetros de cor e atividade de água

KUROISHI, A. M. et al.

armazenamento não houve cristalização, mesmo naquela em que foram adicionados $5 \%$ de cristais (Ensaio 1), que poderiam atuar como agentes indutores de cristalização. Comparando-se os dois ensaios, o Índice de Brancura do Ensaio 1 apresentou valor maior que o do Ensaio 2, reforçando o fato de que a presença de cristais no sistema, mesmo que quase imperceptíveis a olho nu, provoca certa opacidade nas amostras, refletida pelo aumento do IB.

Foi possível observar uma tendência de aumento desse índice nas amostras armazenadas em temperaturas menores, de 11 e $21{ }^{\circ} \mathrm{C}$ (Ensaios 3 e 4 e repetições do ponto central), sendo esse aumento decorrente da formação dos cristais no mel, o que concorda com os resultados obtidos para a Aw.

Observando-se a evolução do IB no tempo, sugere-se que o Ensaio 3 e as repetições do ponto central iniciaram a cristalização a partir da primeira semana de armazenamento, e o Ensaio 4 somente após a segunda semana. O Ensaio 4 foi aquele sem adição de cristais, indicando que a presença dos cristais pode acelerar o processo de cristalização, como era esperado.

A adição de cristais de mel apresenta papel importante na cristalização por aumentar a concentração de glicose. Parte dos cristais se dissolve a $40{ }^{\circ} \mathrm{C}$, aumentando a concentração da fase líquida, sendo que aqueles que não foram dissolvidos atuam como núcleos de cristais, aumentando a taxa de cristalização (ASSIL et al., 1991; CONFORTI et al., 2006; ESCOBEDO et al., 2006).

A Figura 4 apresenta os ensaios após duas semanas de armazenamento. Visualmente, observa-se a ocorrência de opacidade nas amostras em processo de cristalização.

Observa-se na Figura 4 que os ensaios 5, 6, 7 e 8 encontram-se com uma coloração semelhante, mas menos opacos que o ensaio 3; este evidencia uma opacidade decorrente da sua intensa cristalização. Por outro lado, comparando-se os ensaios 1 e 2 , armazenados a $31^{\circ} \mathrm{C}$, observa-se que apesar de ambos apresentarem-se translúcidos, o ensaio 1 parece levemente mais opaco, pela presença de cristais dispersos no meio. Isso foi evidenciado pelo maior valor no IB para esse ensaio.
A Tabela 2 apresenta os resultados do parâmetro de luminosidade $L^{*}$ medido durante o tempo de armazenamento das amostras de mel, bem como a sua variação $\left(\Delta \mathrm{L}^{*}\right)$ após três semanas de armazenamento.

De forma a confirmar o efeito da temperatura, assim como o da adição de cristais sobre a cristalização do mel com o tempo, utilizou-se o $\Delta L^{*}$ (variação do $L^{*}$ com o tempo de 21 dias ou 3 semanas) como variável resposta ao experimento fatorial completo.

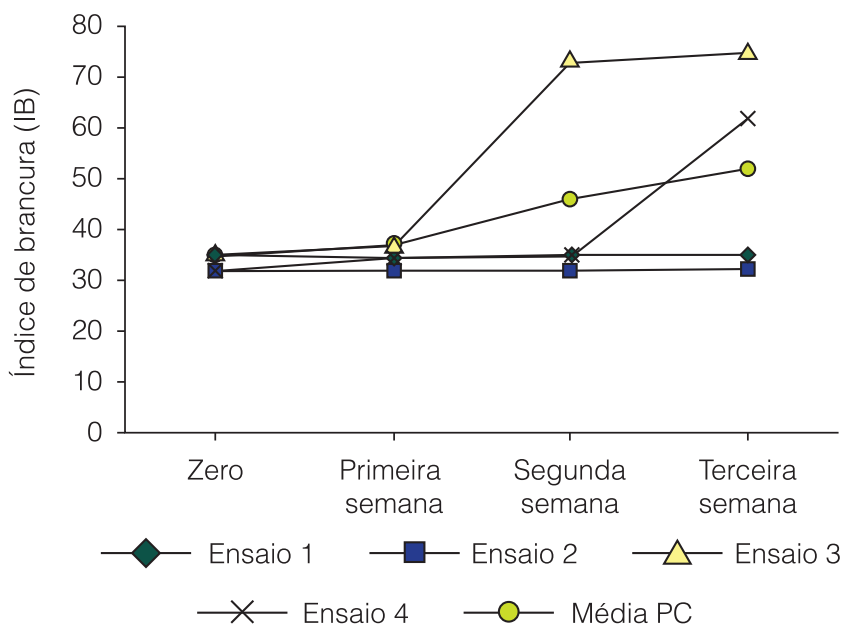

Figura 3. Índice de brancura $\times$ tempo de armazenamento para os ensaios do delineamento.

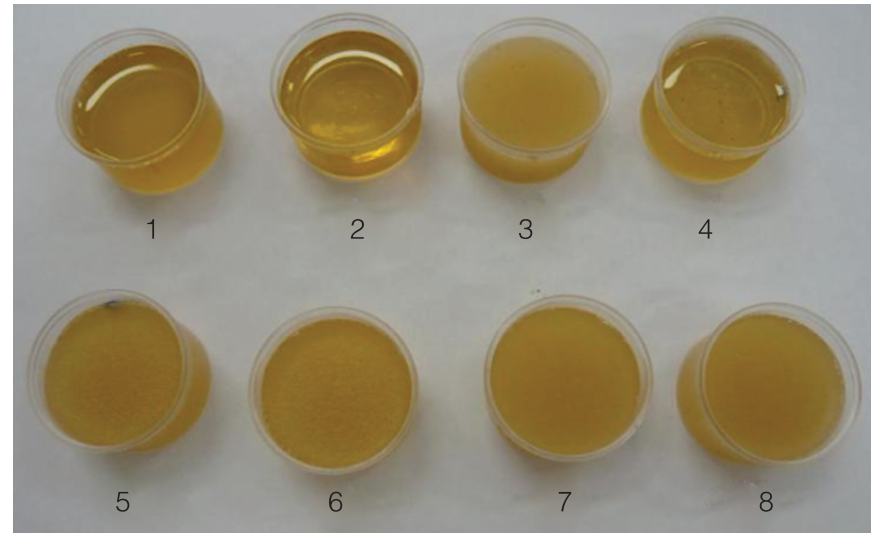

Figura 4. Aparência dos ensaios do delineamento após duas semanas de armazenamento.

Tabela 2. Variação da luminosidade com o tempo.

\begin{tabular}{cccccr} 
Ensaio & zero & $\mathbf{1}^{\mathbf{a}}$ semana & $\mathbf{2}^{\mathbf{a}}$ semana & $\mathbf{3}^{\mathbf{a}}$ semana & \multicolumn{1}{c}{$\mathbf{L}^{*}$} \\
1 & 35,45 & 34,92 & 34,89 & 35,21 & $-0,24$ \\
2 & 31,88 & 31,90 & 31,94 & 32,25 & 0,37 \\
3 & 35,17 & 36,53 & 78,14 & 81,17 & 46,00 \\
4 & 31,88 & 34,91 & 35,21 & 64,46 & 32,58 \\
5 & 35,07 & 35,65 & 46,46 & 52,99 & 17,92 \\
6 & 35,07 & 35,70 & 44,90 & 53,70 & 18,63 \\
7 & 34,92 & 38,03 & 48,36 & 54,29 & 19,37 \\
8 & 34,92 & 38,35 & 46,06 & 52,69 & 17,77 \\
\hline
\end{tabular}


Avaliação da cristalização de mel utilizando parâmetros de cor e atividade de água

KUROISHI, A. M. et al.

A Tabela 3 apresenta os coeficientes de regressão e os valores de $p$ para as variáveis temperatura de armazenamento $\left({ }^{\circ} \mathrm{C}\right)$ e cristais adicionados (\%), obtidos por meio da análise estatística. O nível de significância adotado foi de 95\%. A Tabela 4, por sua vez, apresenta os resultados da Análise de Variância (ANOVA) para as medidas do $\Delta L^{*}$, de acordo com o planejamento estatístico. O coeficiente de regressão para o modelo gerado foi de 0,9966, considerado bom para o experimento realizado.

Visto que o valor de $\mathrm{F}$ calculado para a regressão foi maior que o $\mathrm{F}$ tabelado, o modelo para estimar o $\Delta \mathrm{L}^{*}$

Tabela 3. Valores de p para as variáveis temperatura de armazenamento e percentual de cristais adicionados.

\begin{tabular}{lcc}
\multicolumn{1}{c}{ Variável } & $\begin{array}{c}\text { Coeficiente de } \\
\text { regressão }\end{array}$ & $\begin{array}{c}\text { Valor } \\
\text { de } \mathbf{p ~}\end{array}$ \\
\hline Temperatura $\left({ }^{\circ} \mathrm{C}\right)$ & $-19,49$ & 0,000005 \\
$\%$ Cristais & 3,32 & 0,004626 \\
Temp. $\times$ Crist. $\left({ }^{\circ} \mathrm{C} \times \%\right)$ & $-3,39$ & 0,004311 \\
\hline
\end{tabular}

foi considerado válido e preditivo, e é apresentado na Equação 3.

$y=19,11-19,49 x_{1}+3,32 x_{2}-3,39 x_{1} x_{2}$

em que: $Y=\Delta L^{*} ; \quad x_{1}$ e $x_{2}=$ valores codificados para a temperatura e para o \% de cristais de mel adicionados.

Avaliando-se os principais efeitos da temperatura e do percentual de cristais no parâmetro de luminosidade $\Delta \mathrm{L}^{*}$, observou-se que o efeito exercido pela temperatura é maior quando comparado ao efeito exercido pela adição de cristais e pela interação da temperatura com os cristais.

O efeito das variáveis temperatura e percentual de cristais sobre a variação do parâmetro de luminosidade é apresentado na Figura 5.

Observa-se que os maiores valores de $\Delta \mathrm{L}^{*}$ encontram-se na região com a menor temperatura de armazenamento $\left(11^{\circ} \mathrm{C}\right)$ e com o maior teor de cristais de mel adicionados (5\%). Com a diminuição da temperatura, ocorre a diminuição da solubilidade dos açúcares presentes no mel, favorecendo a sua cristalização.

Tabela 4. Análise de Variância (ANOVA) para o planejamento estatístico.

\begin{tabular}{lrrrrr} 
Fonte de variação & \multicolumn{1}{c}{$\mathbf{S Q}$} & $\mathbf{G L}$ & $\mathbf{M Q}$ & $\mathbf{F}_{\text {calc }}$ & $\mathbf{F}_{\text {tab }}$ \\
Regressão & 1609,887 & 2 & 804,943 & 745,27 & 5,79 \\
Resíduos & 5,400 & 5 & 1,080 & 3,50 & 9,55 \\
Erro puro & 3,781 & 3 & 1,891 & \\
Falta de ajuste & 1,619 & 2 & 0,540 & & \\
Total & 1615,287 & 7 & & \\
\hline
\end{tabular}

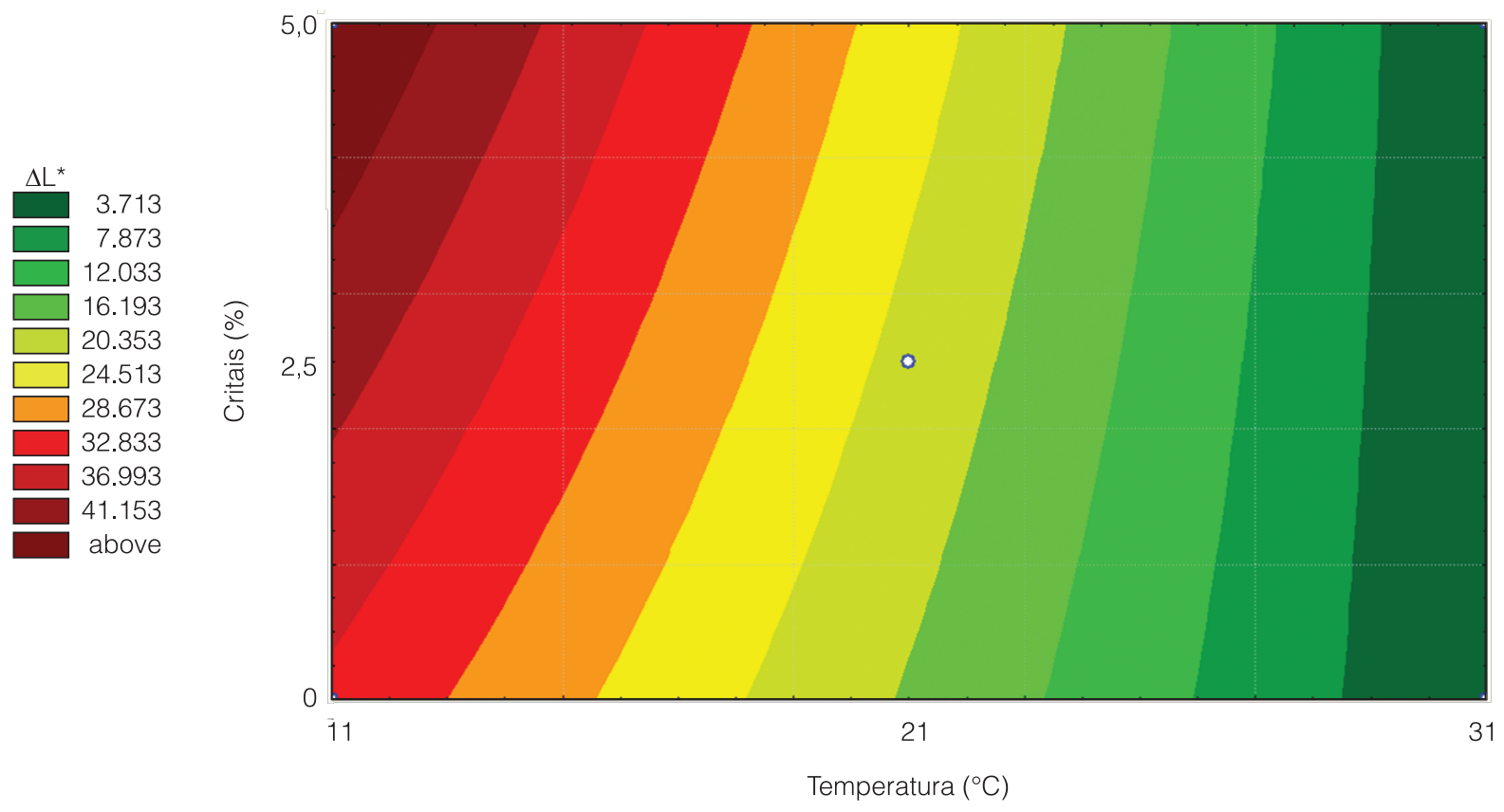

Figura 5. Curva de contorno para temperatura e \% de cristais de mel. 
Avaliação da cristalização de mel utilizando parâmetros de cor e atividade de água

KUROISHI, A. M. et al.

\section{Conclusões}

Foi possível verificar que o Índice de Brancura (IB) aumenta à medida que ocorre a cristalização do mel, indicando que esse índice pode ser usado para o monitoramento da cristalização. Foi observado que a cristalização do mel é influenciada pela temperatura de armazenamento, assim como pelo teor de cristais de mel adicionado; observou-se também que temperaturas maiores ou iguais a $30^{\circ} \mathrm{C}$ tendem a manter a solução do mel em equilíbrio, não favorecendo a sua cristalização.

Como era de se esperar, a adição de cristais em temperaturas mais baixas induz uma cristalização mais rápida do sistema, pois esses cristais servem como "sementes" de cristalização, aumentando a velocidade de sua ocorrência.

O uso da determinação objetiva da cor pelo sistema CIELAB e a conversão dos parâmetros de $L^{*}, a^{*}$ e $b^{*}$ para o Índice de Brancura podem ser uma ferramenta adequada e relativamente simples para o acompanhamento da cinética de cristalização em mel.

\section{Agradecimentos}

Ao CNPq pelo apoio financeiro no projeto: Processo 478936/2010-9

\section{Referências}

ABRAMOVIC, H.; JAMNIK, M.; BURKAN, L.; KAC, M. Water activity and water content in Slovenian honeys. Food Control, Oxford, v. 19, n. 11, p. 1086-1090, 2008. http://dx.doi. org/10.1016/j.foodcont.2007.11.008

ABU-JDAYIL, B.; GHZAWI, A. A. M.; AL-MALAH, K. I. M.; ZAITOUN, S. Heat effect on rheology of light - and dark- colored honey. Journal of Food Engineering, Oxford, v. 51, n. 1, p. 33-38, 2002. http://dx.doi.org/10.1016/S0260-8774(01)00034-6

ASSIL, H. I.; STERLING, R.; SPORNS, P. Crystal control in processed liquid honey. Journal of Food Science, Malden, v. 56, n. 4, p. 1034-1041, 1991. http://dx.doi. org/10.1111/j.1365-2621.1991.tb14635.x

BRASIL. Ministério da Agricultura, Pecuária e Abastecimento. Instrução Normativa $n^{\circ}$ 11, de 20 de outubro de 2000. Regulamento Técnico de identidade e qualidade do mel. Diário Oficial da República Federativa do Brasil, Brasília, DF, 23 out. 2000. Disponível em: <http://extranet.agricultura.gov.br/ sislegis-consulta/servlet/VisualizarAnexo?id=1690>. Acesso em: 06 abr. 2009.

CONFORTI, P. A.; LUPANO, C. E.; MALACALZA, N. H.; ARIAS, V.; CASTELLS, C. B. Crystallization of honey at $-20^{\circ} \mathrm{C}$. International Journal of Food Properties, London, v. 9, n. 1, p. 99-107, 2006. http://dx.doi.org/10.1080/10942910500473962
COSTA, C. C.; PEREIRA, R. G. The influence of propolis on the rheological behaviour of pure honey. Food Chemistry, Oxford, v. 76, n. 4, p. 417-421, 2002. http://dx.doi.org/10.1016/S03088146(01)00298-9

ESCOBEDO, R. M.; ORDÓÑEZ, Y. M.; FLORES, M. E. J.; LÓPEZ, G. F. G. The composition, rheological and thermal properties of Tajonal (Vigueira dentata) Mexican honey. International Journal of Food Properties, London, v. 9, p. 299-316, 2006. http://dx.doi. org/10.1080/10942910600596159

GLEITER, R. A.; HORN, H.; ISENGARD, H. D. Influence of type and state of crystallization on the water activity of honey. Food Chemistry, Oxford, v. 96, n. 3, p. 441-445, 2006. http://dx.doi. org/10.1016/j.foodchem.2005.03.051

GRUNENNVALDT, F. L. Avaliação de Propriedades Físicas e Sensoriais e do Desempenho Tecnológico de Chocolates Produzidos com Misturas de Manteiga de Cacau e Gorduras Low / Zero Trans. 2009. 151 f. Dissertação (Mestrado em Engenharia Química)-Universidade Estadual de Campinas, Campinas, 2009.

INSTITUTO BRASILEIRO DE GEOGRÁFIA E ESTÁTISTICA - IBGE. Produção da Pecuária Municipal. Rio de Janeiro, 2006. v. 34. INSTITUTO BRASILEIRO DE GEOGRÁFIA E ESTÁTISTICA - IBGE. Produção da Pecuária Municipal. Rio de Janeiro, 2009. v. 37.

LAZARIDOU, A.; BILIADERIS, C. G.; BACANDRITSOS, N.; SABATINI, A. G. Composition, thermal and rheological behaviour. Journal of Food Engineering, Oxford, v. 64, n. 1, p. 9-31, 2004. http://dx.doi.org/10.1016/j.jfoodeng.2003.09.007

LENGLER, S. Inspeção e Controle de Qualidade do Mel. Disponível em: <http://www.sebraern.com.br/apicultura/ pesquisas/inspecao_mel01.doc>. Acesso em: 11 jun. 2008.

LUPANO, C. E. DSC study of honey granulation stored at various temperatures. Food Research International, Oxford, v. 30, n. 9, p. 683-688, 1997. http://dx.doi.org/10.1016/S09639969(98)00030-1

MAGALHÃES, E. O. Apicultura - Alternativa de Geração de Emprego e Renda. Disponível em: <http://www.ceplac.gov.br/ radar/Artigos/artigo11.htm>. Acesso em: 20 abr. 2008.

MOREIRA, R. F. A.; DE MARIA, C. A. B. Glicídios no mel. Química Nova, São Paulo, v. 24, n. 4, p. 516-525, 2001.

ROMÃO, R. B. M.; YAMASHITA, F.; BENASSI, M. T.; SOARES, J. H. Metodologia para avaliação de cristalização em balas duras por imagem digitalizada. Brazilian Journal of Food Technology, Campinas, v.9, n.2, p. 151-155, 2006.

SILVA, C. L.; QUEIROZ, A. J. M.; FIGUEIREDO, R. M. F. Caracterização físico-química de méis produzidos no estado do Piauí para diferentes floradas. Revista Brasileira de Engenharia Agrícola e Ambiental, Campinas Grande, v. 8, 
Avaliação da cristalização de mel utilizando parâmetros de cor e atividade de água

KUROISHI, A. M. et al.

n. 2-3, p. 260-265, 2004. http://dx.doi.org/10.1590/S141543662004000200015

SUBRAMANIAN, R.; HEBBAR, H. U.; RASTOGI, N. K. Processing of honey: a review. International Journal of Food Properties, London, v. 10, n. 1, p. 127-143, 2007. http://dx.doi. org/10.1080/10942910600981708

VARGAS, T. Avaliação da Qualidade do Mel Produzido na Região dos Campos Gerais do Paraná. 2006. 134 f. Dissertação (Mestrado em Ciência e Tecnologia de Alimentos)Universidade Estadual de Ponta Grossa, Ponta Grossa, 2006.
WIESE, H. Nova Apicultura. 7. ed. Porto Alegre: Agropecuária, 1986. 493 p.

ZAMORA, M. C.; CHIRIFE, J. Determination of water activity change due to crystallization in honeys from Argentina. Food Control, Oxford, v. 17, n. 1, p. 59-64, 2006. http://dx.doi. org/10.1016/j.foodcont.2004.09.003

ZUMLA, A.; LULAT A. Honey: a remedy rediscovered. Journal of the Royal Society of Medicine, London, v. 82, n. 7, p. 384-385, 1989. 